\title{
Mimetic massive gravity: beyond linear approximation
}

\author{
Ali H. Chamseddine ${ }^{a, b}$ and Viatcheslav Mukhanov ${ }^{c, d, e}$ \\ ${ }^{a}$ Physics Department, American University of Beirut, \\ Beirut, Lebanon \\ ${ }^{b}$ I.H.E.S., \\ F-91440 Bures-sur-Yvette, France \\ ${ }^{c}$ Theoretical Physics, Ludwig Maxmillians University, \\ Theresienstr. 37, 80333 Munich, Germany \\ ${ }^{d}$ MPI for Physics, \\ Foehringer Ring, 6, 80850, Munich, Germany \\ ${ }^{e}$ LPT de l'Ecole Normale Superieure, \\ 24 rue Lhomond, 75231 Paris cedex, France \\ E-mail: chams@aub.edu.1b, viatcheslav.mukhanov@lmu.de
}

ABSTRACT: We present a theory of ghost-free massive gravity where the mass of the graviton is generated through the Brout-Englert-Higgs (BEH) mechanism and one of the four scalar fields used is that of mimetic gravity. The mass term is not of the Fierz-Pauli type and the constraint eliminates the Boulware-Deser ghost which is absent to all orders. We perform a detailed analysis using the methods of cosmological perturbation theory and consider quantum fluctuations of the degrees of freedom of massive graviton and mimetic matter. It is shown that for three of the degrees of freedom of the graviton of mass $m$ the nonlinear corrections become comparable to the linear terms already at a length scale of order $m^{-\frac{1}{2}}$. Thus, at smaller scales they become strongly coupled and the graviton remains with two transverse degrees of freedom which get strongly coupled only at Planck scale. The mimetic field behaves as cold particles of half of the graviton mass and could well explain the source of dark matter in our universe. In the weakly coupled domain mimetic matter is completely decoupled from the massive graviton.

KEYwords: Classical Theories of Gravity, Effective Field Theories

ARXIV EPRINT: 1805.06598 


\section{Contents}

1 Introduction 1

2 BEH-mechanism for massive gravity 2

3 Mimetic non Fierz-Pauli massive gravity 4

4 Graviton on Minkowski background 5

5 Strong coupling and massless limit $\quad 12$

6 Static gravitational field by external source $\quad 14$

$\begin{array}{lll}7 & \text { Summary } & 17\end{array}$

\section{Introduction}

The problem of finding a consistent theory of massive gravity has recently attracted considerable interest. It is by now well established that the simplest way of giving mass to the graviton without explicit breaking of diffeomorphism invariance is by employing four scalar fields, which acquire vacuum expectation values [1-3]. As a result the scalar fields are absorbed making the graviton massive on Minkowski background. However, four scalar fields have generically four degrees of freedom and only three of them are needed to provide mass to the graviton. Out of four scalar fields, used to preserve global Lorentz invariance, one field must be taken with negative kinetic energy and if its perturbations around a spontaneously broken symmetry background propagates then there would appear a ghost mode leading to inconsistency of the theory. By adopting the Fierz-Pauli (FP) term for the mass [4] one can vanish the kinetic term for these perturbations, so that the dangerous mode disappears in the linear approximation. Generically it reappears again and starts to propagate on a nontrivial background and is known as nonlinear Boulware-Deser (BD) ghost [5]. There was recently an attempt to extend the FP-term to higher orders in such a way as to avoid the propagation of this field to all orders on a nontrivial background. The resulting theory which might avoid the non-linear BD-ghost is rather unambiguous and its action is given by an infinite expansion of a square root function [6] which can be simplified and rewritten as a quadratic one by making use of auxiliary fields [7]. However, in some particular backgrounds, the ghost mode nevertheless gets excited, raising a question about consistency of the theory $[8,10]$.

One can wonder whether it is possible to have a ghost-free massive gravity which is not very restricted and well behaved to all higher orders? We will show in this paper that this can be easily achieved in mimetic gravity [9], with non Fierz-Pauli mass term, where 
we use the constrained scalar field imitating Dark Matter as one of the four fields needed to realize Brout-Englert-Higgs (BEH) mechanism for the graviton mass.

\section{BEH-mechanism for massive gravity}

In BEH-mechanism for massive gravity a central role is played by four scalar fields $\phi^{A}\left(x^{\mu}\right)$, $A=0,1,2,3$, which in the broken symmetry phase, in Minkowski space-time, acquire vacuum expectation values

$$
\left\langle\phi^{A}\right\rangle=\delta_{\mu}^{A} x^{\mu} \equiv x^{A}
$$

Note that the vacuum state is degenerate and the different vacua are related by Poicnare transformations. From the auxiliary induced metric $H^{A B}=g^{\mu \nu} \phi_{, \mu}^{A} \phi_{, \nu}^{B}$, where $\phi_{, \mu}^{A} \equiv \frac{\partial \phi^{A}}{\partial x^{\mu}}$, it is convenient to build the diffeomorphism invariant set of scalars

$$
\bar{h}^{A B}=H^{A B}-\eta^{A B},
$$

and use them to give mass to the graviton. Here $\eta^{A B}=(1,-1,-1,-1)$ is the auxiliary Minkowski metric. Let us consider the small perturbations of the scalar fields around broken symmetry phase

$$
\phi^{A}=x^{A}+\chi^{A}
$$

which induce small metric perturbations

$$
g^{\mu \nu}=\eta^{\mu \nu}+h^{\mu \nu} .
$$

Then

$$
\bar{h}^{A B}=h^{A B}+\partial^{A} \chi^{B}+\partial^{B} \chi^{A}+h^{A \nu} \chi_{, \nu}^{B}+h^{B \nu} \chi_{, \nu}^{A}+\eta^{\mu \nu} \chi_{, \mu}^{A} \chi_{, \nu}^{B}+h^{\mu \nu} \chi_{, \mu}^{A} \chi_{, \nu}^{B},
$$

where $h^{A B} \equiv \delta_{\mu}^{A} \delta_{\nu}^{B} h^{\mu \nu}, \partial^{A} \chi^{B} \equiv \eta^{A \nu} \chi_{, \nu}^{B}, \eta^{A \nu} \equiv \delta_{\mu}^{A} \eta^{\mu \nu}, h^{A \nu}=\delta_{\mu}^{A} h^{\mu \nu}$ etc. The scalars $\bar{h}^{A B}$ are diffeomorphism invariant and by an appropriate choice of coordinate system $x^{\mu}$ we can impose four gauge conditions on fourteen functions $\chi^{A}$ and $h^{\mu \nu}$ out of which they are built. For instance, in so called unitary gauge where $\chi^{A}=0, \bar{h}^{A B}$ coincides with the metric perturbations $h^{\mu \nu}$. Thus, it is clear that $\bar{h}^{A B}$ can be used to construct, in a gauge invariant way, the mass term for the graviton via BEH-mechanism. For instance, a theory with the action

$$
S=\int d^{4} x \sqrt{-g}\left[-\frac{1}{2} R+\frac{m^{2}}{8}\left(\bar{h}^{2}-\bar{h}^{A B} \bar{h}_{A B}\right)\right],
$$

where $\bar{h}=\bar{h}_{A}^{A}$ and we use the units in which $8 \pi G=1$, describes massive gravity with FPmass term in broken symmetry phase. Note that contraction of capital indices guarantees the invariance of the theory with respect to a particular choice of the vacuum in a huge landscape of degenerate vacuum states related by Poincare transformations.

One can easily see why the Fierz-Pauli combination is so special by considering a decoupling limit when the metric perturbations $h^{\mu \nu}$ vanish. In this case the mass term

$$
\frac{m^{2}}{8}\left(\alpha \cdot \bar{h}^{2}-\bar{h}^{A B} \bar{h}_{A B}\right),
$$


where $\alpha$ is a numerical coefficient, becomes (up to total derivative)

$$
\frac{m^{2}}{2}\left(-\frac{1}{4} F^{A B} F_{A B}+(\alpha-1)\left(\partial_{A} \chi^{A}\right)^{2}+O\left(\chi^{3}\right)\right)
$$

with $F_{A B}=\partial_{A} \chi_{B}-\partial_{B} \chi_{A}$. It is clear that unless $\alpha=1$, the theory with Lagrangian (2.8) describes four fields one of which is inevitably a ghost. Only the choice of the mass combination $\bar{h}^{2}-\bar{h}^{A B} \bar{h}_{A B}$ insures that the term $\left(\bar{h}_{00}\right)^{2}$, that provides dynamics to the ghost field $\chi^{0}$, is absent in the Lagrangian and $\chi^{0}$ becomes similar to the gauge potential $A^{0}$ in Maxwell theory, which corresponds to the first term in (2.8). However, if one considers the propagation of the fields in a curved background (for instance induced by an external source) with metric $g_{\mu \nu}=\eta_{\mu \nu}+h_{\mu \nu}$, then the contribution

$$
\bar{h}^{2}=2 \bar{h}_{i}^{i} \bar{h}_{0}^{0}+\ldots=2 h_{i}^{i}\left(\dot{\chi}^{0}\right)^{2}+\ldots,
$$

due to the higher order terms in (2.5) induces a propagator for $\chi^{0}$, thus resurrecting the nonlinear BD-ghost in non-trivial background even for FP mass term. One can try to get rid of the nonlinear ghost by adding to the action (2.6) higher order terms $\bar{h}^{3}, \bar{h} \bar{h}^{A B} \bar{h}_{A B}, \ldots$ and such strategy leads to a nearly unambiguous theory with action [6]:

$$
S=\int d^{4} x \sqrt{g}\left[-\frac{1}{2} R+\frac{m^{2}}{2}\left(S^{2}-S^{A B} S_{A B}\right)\right]
$$

where

$$
S_{A B}=\sqrt{\eta_{A B}+\bar{h}_{A B}}-\eta_{A B}
$$

To second order in $\bar{h}$ this theory reduces to FP theory. In higher orders it is represented by an infinite series in $\bar{h}$ and only with the help of auxiliary vierbein type fields the square root in (2.11) can be given in finite form and the theory becomes quadratic in $S$ [7]. The theory (2.10) looks promising from the point of view of keeping the field $\chi^{0}$ non-dynamical to all orders. However, it was shown in $[7,8]$ that the term $\bar{h}^{0 i} \bar{h}_{0 i}$, which is already present in FP-term, as part of the quadratic term $h^{A \nu} \chi_{, \nu}^{B}$ in (2.5), gives the following contribution to the action

$$
\bar{h}^{0 i} \bar{h}_{0 i}=h^{0 i} h^{0 i}\left(\dot{\chi}^{0}\right)^{2}+\ldots
$$

which is not canceled by higher order terms. Thus, in some backgrounds the field $\chi^{0}$ starts to propagate again and there is no guarantee that this mode would not induce a ghost in an arbitrary background. A reader could wonder whether it is possible to choose a gauge $h^{0 i}=0$ where the term $(2.12)$ is absent. It was shown in $[8,10]$ that in this gauge the propagating $\chi^{0}$ mode reappears in a more complicated way. This is why the question about ghost free massive gravity remains yet open. In this paper we address the following questions:

- Is it in principle possible to construct ghost free theory which will describe massive graviton with five degrees of freedom in Minkowski background?

- Must the FP mass term be necessarily always used in massive gravity? 


\section{Mimetic non Fierz-Pauli massive gravity}

As we have seen above the problem in massive gravity is that the field $\phi^{0}$ becomes dynamical by itself and thus inevitably leads to the appearance of linear or nonlinear ghost. The idea we exploit in this paper is to use in BEH-mechanism the mimetic field [9] as one of four fields responsible for the appearance of the graviton mass. This field $\phi^{0}$ always obeys the constraint

$$
g^{\mu \nu} \partial_{\mu} \phi^{0} \partial_{\nu} \phi^{0}=1
$$

and in a synchronous coordinate system serves as time coordinate, so that the generic solution of (3.1) is

$$
\phi^{0}=t+A
$$

where $A$ is a constant of integration. Combined with the longitudinal mode of gravity it leads to the appearance of mimetic matter which can well imitate the observed Dark Matter in the Universe [9, 11]. The remaining three scalar fields can provide three extra degree of freedom needed for the massive graviton. Thus, in such theory we are guaranteed to have no ghosts to any order in perturbation theory. It happens that in this case the mass term for the graviton is unambiguously fixed. It is not of the Fierz-Pauli type and the action of the corresponding theory is

$$
S=\int d^{4} x \sqrt{g}\left[-\frac{1}{2} R+\frac{m^{2}}{8}\left(\frac{1}{2} \bar{h}^{2}-\bar{h}^{A B} \bar{h}_{A B}\right)+\lambda\left(g^{\mu \nu} \partial_{\mu} \phi^{0} \partial_{\nu} \phi^{0}-1\right)\right] .
$$

The mass term has a relative coefficient of $-\frac{1}{2}$ between the $\bar{h}^{2}$ and $\bar{h}^{A B} \bar{h}_{A B}$ terms. The mimetic constraint term does not violate the Lorentz invariance of the ground state because it is obviously invariant with respect to the transformation $\phi^{0} \rightarrow \tilde{\phi}^{0}=\Lambda_{B}^{0} \phi^{B}$ for $\phi^{B}=$ $\delta_{\mu}^{B} x^{\mu}$. Variation of this action with respect to the metric $g_{\mu \nu}$ gives the following modified Einstein equations

$$
\begin{aligned}
G_{\nu}^{\mu}= & 2 \lambda \partial^{\mu} \phi^{0} \partial_{\nu} \phi^{0}-\frac{m^{2}}{8}\left(\frac{1}{2} \bar{h}^{2}-\bar{h}^{A B} \bar{h}_{A B}\right) \delta_{\nu}^{\mu} \\
& +\frac{m^{2}}{2}\left(\frac{1}{2} \bar{h} \partial^{\mu} \phi_{A} \partial_{\nu} \phi^{A}-\bar{h}_{B}^{A} \partial^{\mu} \phi_{A} \partial_{\nu} \phi^{B}\right),
\end{aligned}
$$

where $G_{\nu}^{\mu}=R_{\nu}^{\mu}-\frac{1}{2} R \delta_{\nu}^{\mu}$ is the Einstein tensor and we recall that capital indices are raised and lowered with the help of the auxiliary metric $\eta_{A B}$, while for Greek indices the spacetime metric $g_{\mu \nu}$ is used. Variation with respect to the scalar fields $\phi_{A}$ leads to

$$
\partial_{\mu}\left(\sqrt{g}\left[\frac{m^{2}}{2}\left(\frac{1}{2} \bar{h} \partial^{\mu} \phi^{A}-\bar{h}_{B}^{A} \partial^{\mu} \phi^{B}\right)+2 \lambda \delta_{0}^{A} \partial^{\mu} \phi^{0}\right]\right)=0 .
$$

Finally the constraint (3.1), which follows by varying with respect to $\lambda$, takes the simple form

$$
\bar{h}_{0}^{0}=0 .
$$

In the next section we will analyze linear perturbations in this model in component form, using the methods of cosmological perturbation theory [12]. An explicitly covariant study of these perturbations is given in a separate publication [13]. 


\section{Graviton on Minkowski background}

We first study the linearized theory considering small metric perturbations in Minkowski background, that is, assuming

$$
g_{\mu \nu}=\eta_{\mu \nu}+h_{\mu \nu},
$$

and $\left|h_{\mu \nu}\right| \ll 1$. Correspondingly the scalar fields deviate from their vacuum expectations values by $\chi^{A}$, that is,

$$
\phi^{A}=x^{A}+\chi^{A} .
$$

To explicitly reveal the true physical degrees of freedom of the massive graviton it is convenient to use the methods of cosmological perturbation theory and classify the perturbations with respect to irreducible representations of the spatial rotation group [12]. The metric component $h_{00}$ behaves as a scalar under rotations and it is convenient to denote it as

$$
h_{00}=2 \phi,
$$

where $\phi$ is a 3 -scalar. The space-time components $h_{0 i}$ behaves as a 3 -vector that can be written as a sum of a longitudinal and transverse parts:

$$
h_{0 i}=B_{, i}+S_{i}
$$

where $B_{, i}=\frac{\partial B}{\partial x^{i}}$ and $S_{i}$ has zero divergence, that is, $\partial^{i} S_{i}=0$. Finally $h_{i j}$ can be decomposed as

$$
h_{i j}=2 \psi \delta_{i j}+2 E_{, i j}+F_{i, j}+F_{j, i}+\tilde{h}_{i j},
$$

with $\partial^{i} F_{i}=0$ and the transverse traceless part $\tilde{h}_{i j}$ satisfies four conditions $\partial^{i} \tilde{h}_{i j}=0$, $\tilde{h}_{i}^{i}=0$, leaving us with two polarizations for the massless graviton in General Relativity. Thus, the perturbations can be classified as scalar perturbations described by $\phi, \psi, B$, and $E$, vector perturbations corresponding to $S_{i}$ and $F_{i}$ and tensor perturbations $\tilde{h}_{i j}$. In the linear approximation they are completely decoupled and thus can be studied separately. In General Relativity in empty space the scalar and vector perturbations vanish and they are induced entirely by matter. In our theory, which can be treated as Einstein theory with extra scalar fields, these perturbations are due to the small perturbations $\chi^{A}$, which in turn can be decomposed as:

$$
\chi^{0}=\chi^{0}, \quad \chi^{i}=\tilde{\chi}^{i}+\pi_{, i}
$$

where $\partial_{i} \tilde{\chi}^{i}=0$. It is obvious that $\chi^{0}$ and $\pi$ excite scalar modes, while $\tilde{\chi}^{i}$ is responsible for vector perturbations. The remaining coordinate freedom

$$
x^{0} \rightarrow x^{0}+\xi^{0}, \quad x^{i} \rightarrow x^{i}+\xi^{i}=x^{i}+\zeta_{, i}+\tilde{\xi}^{i}
$$

with $\partial_{i} \tilde{\xi}^{i}=0$, allows us to impose four gauge conditions. Two of them, which are due to the freedom in choosing $\xi^{0}$ and $\zeta$ refer to the scalar perturbations sector, where for instance two out of seven functions $\phi, \psi, B, E, \chi^{0}, \pi$ and $\lambda$ can be taken to vanish. Often it is very convenient to impose the Newtonian gauge conditions $B=E=0$, which selects 
the coordinate system with explicit Newtonian limit of General Relativity. The coordinate freedom due to transverse $\tilde{\xi}^{i}$ leave the gauge freedom for the vector perturbations and we can set either $S_{i}$ or $F_{i}$ to be equal to zero. The transverse traceless part of the metric $\tilde{h}_{i j}$ is gauge invariant.

Keeping in equations (3.4) only those terms which contain the first order terms in perturbations, these equations are simplified to

$$
\begin{aligned}
G_{0}^{0} & \simeq 2 \lambda+\frac{m^{2}}{4} \bar{h}_{l}^{l}, \\
G_{i}^{0} & \simeq-\frac{m^{2}}{2} \bar{h}_{i}^{0}, \\
G_{k}^{i} & \simeq \frac{m^{2}}{2}\left(\frac{1}{2} \bar{h}_{l}^{l} \delta_{k}^{i}-\bar{h}_{k}^{i}\right),
\end{aligned}
$$

where we took into account that $\lambda$, which is due to mimetic matter, is a first order quantity in perturbations and $\bar{h}_{0}^{0}=0$ thanks to constraint (3.6).

Scalar perturbations. We fix the gauge by taking $B=E=0$. In this gauge the metric takes the form

$$
d s^{2}=(1+2 \phi) d t^{2}-(1-2 \psi) \delta_{i k} d x^{i} d x^{k}
$$

where $\phi$ is the Newtonian gravitational potential. As follows from (2.5) we have to first order in perturbations

$$
\bar{h}_{0}^{0}=-2 \phi+2 \dot{\chi}^{0}, \quad \bar{h}_{i}^{0}=\chi_{, i}^{0}-\dot{\pi}_{, i}, \quad \bar{h}_{k}^{i}=2 \psi \delta_{k}^{i}+2 \pi_{, i j},
$$

where dot denotes derivative with respect to time $t$. Taking into account that in this gauge (see, $[12])$ :

$$
G_{0}^{0}=2 \Delta \psi, G_{i}^{0}=2 \dot{\psi}_{, i}, G_{k}^{i}=(\phi-\psi)_{, i k}-(2 \ddot{\psi}+\Delta(\phi-\psi)) \delta_{i k},
$$

and substituting the expressions above into (4.8)-(4.10) we obtain

$$
\begin{aligned}
\Delta \psi & =\lambda+\frac{m^{2}}{4}(3 \psi+\Delta \pi), \\
\dot{\psi} & =-\frac{m^{2}}{4}\left(\chi^{0}-\dot{\pi}\right),
\end{aligned}
$$

from $0-0$ and $0-i$ equations. The off diagonal $i \neq k$ components of (4.10) give

$$
\phi-\psi=-m^{2} \pi,
$$

while taking the trace we obtain

$$
3 \ddot{\psi}+\Delta(\phi-\psi)+\frac{m^{2}}{4}(3 \psi+\Delta \pi)=0 .
$$

The constraint equation (3.6), when linearized, takes the form

$$
\phi=\dot{\chi}^{0},
$$


and together with equations (4.14)-(4.17) are enough to determine the five unknown variables $\phi, \psi, \lambda, \chi^{0}$ and $\pi$. Differentiating (4.15) and using (4.18) and (4.16) we have

$$
\ddot{\psi}=-\frac{m^{2}}{4}\left(\dot{\chi}^{0}-\ddot{\pi}\right)=-\frac{m^{2}}{4}\left(\psi-m^{2} \pi-\ddot{\pi}\right) .
$$

Substituting this expression in (4.17) and taking into account (4.16) we derive the closed form equation for $\pi$ :

$$
\ddot{\pi}-\Delta \pi+m^{2} \pi=0 .
$$

Considering a plane-wave with wave-number $\vec{k}$ the solution of this equation is

$$
\pi_{k}=\left(A_{k} \sin \omega t+B_{k} \cos \omega t\right) e^{i \vec{k} \cdot \vec{x}}, \quad \omega^{2}=k^{2}+m^{2},
$$

where $A_{k}$ and $B_{k}$ are constants of integration. Using (4.20) we can rewrite (4.19) as

$$
\ddot{\psi}+\frac{m^{2}}{4} \psi=\frac{m^{2}}{4} \Delta \pi
$$

and substituting (4.21) in the right hand side of this equation we solve it to obtain

$$
\psi=C\left(x^{i}\right) \sin \frac{m t}{2}+D\left(x^{i}\right) \cos \frac{m t}{2}+\frac{m^{2} k^{2}}{4 k^{2}+3 m^{2}}\left(A_{k} \sin \omega t+B_{k} \cos \omega t\right) e^{i \vec{k} \cdot \vec{x}},
$$

where $C\left(x^{i}\right)$ and $D\left(x^{i}\right)$ are further space dependent constants of integration describing the contribution of mimetic matter to the gravitational potential $\psi$. It then follows from (4.16) that

$$
\begin{aligned}
\phi & =\psi-m^{2} \pi \\
& =C\left(x^{i}\right) \sin \frac{m t}{2}+D\left(x^{i}\right) \cos \frac{m t}{2}-\frac{3 m^{2} \omega^{2}}{4 k^{2}+3 m^{2}}\left(A_{k} \sin \omega t+B_{k} \cos \omega t\right) e^{i \vec{k} \cdot \vec{x}} .
\end{aligned}
$$

From equation (4.15) one obtains

$$
\begin{aligned}
\chi^{0} & =\dot{\pi}-\frac{4}{m^{2}} \dot{\psi} \\
& =-\frac{2}{m}\left(C\left(x^{i}\right) \cos \frac{m t}{2}-D\left(x^{i}\right) \sin \frac{m t}{2}\right)+\frac{3 m^{2} \omega}{4 k^{2}+3 m^{2}}\left(A_{k} \cos \omega t-B_{k} \sin \omega t\right) e^{i \vec{k} \cdot \vec{x}} .
\end{aligned}
$$

Finally we solve for $\lambda$ from equation (4.14) to get

$$
\lambda=\left(\triangle-\frac{3 m^{2}}{4}\right)\left(C\left(x^{i}\right) \sin \frac{m t}{2}+D\left(x^{i}\right) \cos \frac{m t}{2}\right)
$$

Having determined that the scalar mode of massive graviton is represented by the field $\pi$ satisfying (4.20), we note that $\lambda$ is entirely decoupled from $\pi$ and obeys the equation

$$
\ddot{\lambda}+\frac{m^{2}}{4} \lambda=0
$$


which describes massive mimetic matter. The contribution of this mimetic matter to the gravitational potentials is

$$
\phi=\psi=C\left(x^{i}\right) \sin \frac{m t}{2}+D\left(x^{i}\right) \cos \frac{m t}{2} .
$$

To find the normalized independent quantization variables we need to calculate the action for the scalar perturbations. Let us first expand the action (3.3) to second order in scalar perturbations in the conformal Newtonian gauge, where $B=E=0$,

$$
\begin{aligned}
S=\int d^{4} x[-3 & \dot{\psi}^{2}-\psi \triangle \psi+2 \phi \triangle \psi+2 \lambda\left(\dot{\chi}^{0}-\phi\right) \\
& -\frac{m^{2}}{4}\left(\dot{\chi}^{0}-\phi\right)^{2}+\frac{m^{2}}{2}\left(\dot{\chi}^{0}-\phi\right)(3 \psi+2 \triangle \pi) \\
& \left.+\frac{m^{2}}{4}\left(3 \psi^{2}+2 \psi \triangle \pi-\triangle \pi \triangle \pi\right)+\frac{m^{2}}{4}\left(\chi^{0}-\dot{\pi}\right)_{, i}\left(\chi^{0}-\dot{\pi}\right)_{, i}\right]
\end{aligned}
$$

Variation of this action with respect to $\lambda$ gives the constraint $\phi=\dot{\chi}^{0}$, which when substituted in the action (4.29) reduces it to

$$
S=\int d^{4} x\left[-3 \dot{\psi}^{2}-\psi \triangle \psi+2 \dot{\chi}^{0} \triangle \psi+\frac{m^{2}}{4}\left(3 \psi^{2}+2 \psi \triangle \pi-\triangle \pi \triangle \pi+\left(\chi^{0}-\dot{\pi}\right)_{, i}\left(\chi^{0}-\dot{\pi}\right)_{, i}\right)\right]
$$

Next, as a result of variation with respect to $\chi^{0}$ we obtain the constraint (4.15), and this allows us to express $\chi^{0}$ and $\dot{\chi}^{0}$ in terms of $\psi$ and $\pi$,

$$
S=\int d^{4} x\left[\dot{\psi}\left(-3+\frac{4 \triangle}{m^{2}}\right) \dot{\psi}-\psi \triangle \psi-2 \dot{\pi} \triangle \dot{\psi}+\frac{m^{2}}{4}\left(3 \psi^{2}+2 \psi \triangle \pi-\triangle \pi \triangle \pi\right)\right]
$$

The fields $\psi$ and $\pi$ have mixed propagators. To diagonalize this action we substitute

$$
\psi=\left(\triangle-\frac{3 m^{2}}{4}\right)^{-1}\left(\lambda+\frac{m^{2}}{4} \triangle \pi\right),
$$

that follows from $0-0$ equation (4.14). After some algebra one finds that the action separates in terms of $\pi$ and $\lambda$,

$$
\begin{aligned}
S= & -\frac{1}{2} \int d^{4} x \triangle \pi\left(\frac{2 m^{2}}{3 m^{2}-4 \triangle}\right)\left(\partial_{0}^{2}-\triangle+m^{2}\right) \triangle \pi \\
& +\frac{1}{2} \int d^{4} x \lambda\left(\frac{32}{m^{2}\left(3 m^{2}-4 \triangle\right)}\right)\left(\partial_{0}^{2}+\frac{m^{2}}{4}\right) \lambda,
\end{aligned}
$$

where one should understand $\triangle$ to be $-k^{2}$ for the plane-wave modes with the wave-number $\vec{k}$. This shows that the correctly normalized modes of the corresponding quantum fields are

$$
\sqrt{\frac{2 m^{2}}{3 m^{2}+4 k^{2}}} k^{2} \pi_{k}, \quad \sqrt{\frac{32}{m^{2}\left(3 m^{2}+4 k^{2}\right)}} \lambda_{k}
$$


for the graviton and mimetic matter. The typical amplitude of minimal quantum fluctuations of the properly normalized fields (4.34) in scales $L \sim \frac{1}{k}$ is of order $\left(\frac{k^{3}}{\omega_{k}}\right)^{\frac{1}{2}}$ (see, for instance, formula (4.34) in [14]). Therefore, taking into account that $\omega_{k}=\sqrt{k^{2}+m^{2}}$ for $\pi$ mode and $\omega_{k}=\frac{1}{2} m$ for $\lambda$ mode we infer from (4.34) that the minimal level of quantum fluctuations in scales $L \ll m^{-1}$ for $\pi$ and $\lambda$ are

$$
\delta \pi_{L} \simeq m^{-1}, \quad \delta \lambda_{L} \simeq\left(\frac{m}{L^{5}}\right)^{\frac{1}{2}} .
$$

As it follows from (4.32) and (4.16) they induce the corresponding metric perturbations of order

$$
\delta \phi_{L} \sim \delta \psi_{L} \simeq m^{2} \delta \pi_{L} \simeq m
$$

and

$$
\delta \phi_{L}=\delta \psi_{L} \simeq L^{2} \delta \lambda_{L} \simeq\left(\frac{m}{L}\right)^{\frac{1}{2}},
$$

for the scalar graviton mode and mimetic matter, respectively. Thus the metric perturbations due to the quantum fluctuations of the scalar modes always remain much smaller than unity up to the Planckian scales. Note that we work in Planck units where all constants are set to unity and in dimensional units the right hand side, for instance in (4.37), is $\left(\frac{m}{m_{\mathrm{Pl}}}\right)^{\frac{1}{2}}\left(\frac{l_{\mathrm{Pl}}}{L}\right)^{\frac{1}{2}}$, where $m_{\mathrm{Pl}}$ and $l_{\mathrm{Pl}}$ are the Planck mass and Planck length respectively.

The sign of kinetic energy for the field $\pi$ is positive and this field is not a ghost, while, as follows from (4.33), the apparent contribution to the energy density of mimetic field from the mode with $k \gg m$,

$$
-\frac{4}{m^{2} k^{2}}\left(\dot{\lambda}^{2}+\frac{m^{2}}{4} \lambda^{2}\right)
$$

is negative and looks singular as $m^{2} \rightarrow 0$. However, a propagator for $\lambda$ does not include a Laplacian. Therefore, $\dot{\lambda} \propto m \lambda$, and hence the singularity $\frac{1}{m^{2}}$ in (4.38) is canceled. Moreover, for the mimetic matter the main contribution to the energy density is linear in $\lambda$, so that the total energy density is

$$
\varepsilon_{\operatorname{mim}} \simeq \lambda-\frac{\lambda^{2}}{k^{2}}
$$

where the second negative term just account for the negative contribution of gravitational self-interaction to the total energy density. This second term is smaller than the first term for $\lambda \ll k^{2}$. When $\lambda$ becomes of order $k^{2}$ then we immediately see from (4.34) that $\psi$ becomes of order unity and linear perturbation theory breaks down. To clarify the situation further let us consider in Minkowski space a ball of radius $R$ filled by dust at rest with local energy density $\lambda$. Then the gravitational potential far away from the ball is determined by the mass of the ball which in the leading order can be estimated as $M_{0} \simeq \lambda R^{3}$. However, in the next order, the negative gravitational energy of self interaction of matter within the ball, which is of order $-\frac{M_{0}^{2}}{R}$, also contributes to the mass observed far away from the ball. The resulting mass measured by a far away observer is

$$
M_{\mathrm{obs}} \simeq M_{0}-\frac{M_{0}^{2}}{R} .
$$


If the observer would forget about contribution of negative gravitational energy to the total mass he would conclude that the energy density within the ball is

$$
\varepsilon_{\mathrm{obs}} \simeq \frac{M_{\mathrm{obs}}}{R^{3}} \simeq \lambda-R^{2} \lambda^{2} .
$$

Taking into account that $k \simeq R^{-1}$ this explains the origin of negative energy contribution to the mimetic matter energy density. When the second term in (4.41) becomes comparable with the first one we first get semi-closed worlds and finally when the negative self-interaction energy exactly compensates the internal mass one obtains the closed universe with zero total energy with respect to an observer in asymptotically Minkowski space (see, for example, [15, page 109] for details).

Vector perturbations. Let us now turn to vector perturbations taking the gauge $F_{i}=0$, so that the metric becomes

$$
d s^{2}=d t^{2}+2 S_{i} d x^{i} d t-\delta_{i k} d x^{i} d x^{k} .
$$

As follows from (2.5), (4.5) and (4.6), we have, to linear order in perturbations,

$$
\bar{h}_{0}^{0}=0, \quad \bar{h}_{i}^{0}=-S_{i}+\dot{\tilde{\chi}}_{i}, \quad \bar{h}_{k}^{i}=-\tilde{\chi}_{k, i}-\tilde{\chi}_{i, k},
$$

where as we recall $\tilde{\chi}_{i}=-\tilde{\chi}^{i}$ and both $S_{i}$ and $\tilde{\chi}_{i}$ are transverse. Taking into account that in the gauge $F_{i}=0$ :

$$
G_{0}^{0}=0, \quad G_{i}^{0}=\frac{1}{2} \Delta S_{i}, \quad G_{k}^{i}=-\frac{1}{2}\left(\dot{S}_{i, k}+\dot{S}_{k, i}\right),
$$

(see, [12]) equations (4.9) and (4.10) reduce to

$$
\begin{aligned}
\Delta S_{i} & =m^{2}\left(S_{i}-\dot{\tilde{\chi}}_{i}\right), \\
\dot{S}_{i, k}+\dot{S}_{k, i} & =-m^{2}\left(\tilde{\chi}_{i, k}+\tilde{\chi}_{k, i}\right),
\end{aligned}
$$

while equation (4.8) is satisfied identically. As follows from (4.45)

$$
S_{i}=\frac{m^{2}}{m^{2}-\Delta} \dot{\tilde{\chi}}_{i}
$$

and this, after being substituted in (4.46), gives

$$
\left(\ddot{\tilde{\chi}}_{i, k}+\ddot{\tilde{\chi}}_{k, i}\right)-\Delta\left(\tilde{\chi}_{i, k}+\tilde{\chi}_{k, i}\right)+m^{2}\left(\tilde{\chi}_{i, k}+\tilde{\chi}_{k, i}\right)=0,
$$

or, equivalently,

$$
\ddot{\tilde{\chi}}_{i}-\Delta \tilde{\chi}_{i}+m^{2} \tilde{\chi}_{i}=0 .
$$

This equation describes two vector modes of the massive graviton. The action for the vector modes can be obtained by expanding (3.3) to second order in perturbations:

$$
S=\frac{1}{4} \int d^{4} x\left[S_{i, k} S_{i, k}+m^{2}\left(\left(\dot{\tilde{\chi}}_{i}-S_{i}\right)\left(\dot{\tilde{\chi}}_{i}-S_{i}\right)-\tilde{\chi}_{i, k} \tilde{\chi}_{i, k}\right)\right] .
$$


Variation of this action with respect to $S_{i}$ gives us constraint equation (4.45), and upon substituting (4.47), the action reduces to

$$
S=-\frac{1}{2} \int d^{4} x\left[\tilde{\chi}_{i}\left(\frac{m^{2} \Delta}{2\left(\Delta-m^{2}\right)}\right)\left(\partial_{0}^{2}-\Delta+m^{2}\right) \tilde{\chi}_{i}\right] .
$$

From this we deduce that the properly normalized modes for the vector perturbations are

$$
\sqrt{\frac{m^{2} \Delta}{2\left(\Delta-m^{2}\right)}} \tilde{\chi}_{i} .
$$

Thus for plane-wave perturbations with momentum $k \simeq L^{-1} \gg m$ the typical minimal quantum fluctuations in scales $L$ is of order

$$
\delta \tilde{\chi}_{L} \simeq \frac{1}{m L} .
$$

Taking into account that $\delta \dot{\tilde{\chi}}_{L} \simeq \frac{\delta \tilde{\chi}_{L}}{L}$ we infer from (4.47) that the corresponding metric perturbations are

$$
\delta S_{i L} \simeq m
$$

Tensor perturbations. The tensor perturbations $\tilde{h}_{i j}$ satisfy four extra conditions $\partial^{i} \tilde{h}_{i j}=0=\tilde{h}_{i}^{i}$. They are gauge invariant and describe two degree of freedom of the graviton which have become massive. The equation for $\tilde{h}_{i j}$ immediately follows from (4.10),

$$
\left(\partial_{0}^{2}-\Delta+m^{2}\right) \tilde{h}_{i j}=0
$$

and the corresponding action is

$$
S=-\frac{1}{8} \int d^{4} x\left[\tilde{h}_{i j}\left(\partial_{0}^{2}-\Delta+m^{2}\right) \tilde{h}_{i j}\right] .
$$

The canonical quantization variable is $\tilde{h}_{i j}$ and hence the typical amplitude of quantum fluctuations for tensor modes in scales $L$ is of order

$$
\delta \tilde{h}_{i j} \simeq \frac{1}{L},
$$

for $L \ll m^{-1}$. They become of order unity at Planck scale $l_{\mathrm{Pl}} \simeq 10^{-33} \mathrm{~cm}$ where they enter non-perturbative quantum gravity strong coupling regime and linearized theory ceases to be applicable. Although the amplitude of quantum scalar and vector metric perturbations is scale independent $\delta \psi_{L} \simeq \delta S_{L} \simeq m$ and remains small even at the Planck scale, these perturbations nevertheless enter the strong coupling regime at the energy scale which is much below the Planck scale, that is, well before the tensor modes (see next section).

Thus we have shown in this section that the theory with action (3.3) describes on a Minkowski background a massive graviton with five degrees of freedom. According to little (rotation) group representations they can be thought of as consisting of one scalar degree of freedom described by $\pi$, two vector degrees of freedom $\tilde{\chi}_{i}$ and two tensor modes $\tilde{h}_{i j}$. In addition we have a massive mimetic matter described by $\lambda$ which, in the linear approximation, completely decouples from the massive graviton. These results are valid only when the higher order corrections to the linearized equation are negligible. In the next section we determine the range of applicability of the linearized theory. 


\section{$5 \quad$ Strong coupling and massless limit}

Scalar modes. To determine at which scales the nonlinear corrections to the linearized equations become important we need to calculate the next order terms in equations (3.4). Because the resulting expressions are too cumbersome even to second order, we will keep in the formulae only those terms which can become comparable to linear terms assuming that the gravitational potentials $\phi$ and $\psi$ are much smaller than unity and considering perturbations with $k^{2} \gg m^{2}$. In the left hand side of equations (3.4) it is enough to keep only the linear terms because under the assumptions stated above all terms of order $\phi^{2}$, $\psi^{2}$ are small compared to the linear terms. Keeping in $\bar{h}_{0}^{0}$, defined in (2.5), only terms to second order in perturbations we have

$$
0=\bar{h}_{0}^{0}=-2\left(\phi-\dot{\chi}^{0}\right)+4\left(\phi-\frac{1}{2} \dot{\chi}^{0}\right)^{2}-\chi_{, i}^{0} \chi_{, i}^{0}
$$

It is clear that $O\left(\phi^{2}\right)$ terms are always much smaller than $\phi$ and can be skipped. The term $4 \phi \dot{\chi}^{0}$ is much smaller than $\dot{\chi}^{0}$ for $\phi \ll 1$ and hence can be neglected in $\bar{h}_{0}^{0}$. Therefore, keeping only relevant terms to second order in perturbations, the constraint $\bar{h}_{0}^{0}=0$ becomes

$$
-2 \phi+2 \dot{\chi}^{0}+\left(\dot{\chi}^{0}\right)^{2}-\chi_{, i}^{0} \chi_{, i}^{0} \simeq 0
$$

For linear perturbations we can express $\phi, \psi$ and $\chi^{0}$ in terms of the independent fields $\pi$ and $\lambda$ using (4.21)-(4.26):

$$
\begin{aligned}
\phi=\dot{\chi}^{0} & =-\frac{3 m^{2} \omega^{2} \pi+4 \lambda}{4 k^{2}+3 m^{2}} \simeq-\frac{3 m^{2}}{4} \pi-\frac{\lambda}{k^{2}}, \\
\psi & =\frac{m^{2} k^{2} \pi-4 \lambda}{4 k^{2}+3 m^{2}} \simeq \frac{m^{2}}{4} \pi-\frac{\lambda}{k^{2}}, \\
\chi^{0} & =\frac{3 m^{4} \dot{\pi}+16 \dot{\lambda}}{m^{2}\left(4 k^{2}+3 m^{2}\right)} \simeq \frac{3 m^{2}}{4 k} \pi+\frac{2}{m k^{2}} \lambda,
\end{aligned}
$$

where to simplify the formulae in the second equality we considered perturbations with scales $L \simeq k^{-1} \ll m^{-1}$ and therefore skipped all subleading corrections proportional to $\frac{m^{2}}{k^{2}}$. Moreover in (5.5) we estimated the time derivatives as $\dot{\lambda} \simeq \frac{m}{2} \lambda$ and $\dot{\pi} \simeq k \pi$. It is clear that the $\left(\dot{\chi}^{0}\right)^{2}$ term in (5.2) is of order $\phi^{2}$ and can be neglected compared to the last term, which in the domain of applicability of the linear theory would signal us when the linearized equations fail. Namely, this happens at scales where $\chi_{, i}^{0} \chi_{, i}^{0} \sim \frac{\left(\chi^{0}\right)^{2}}{L^{2}}$ becomes comparable with $\phi$ given in (5.3). The contributions of quantum fluctuations to the gravitational potential from the scalar mode of the graviton $\pi$ and mimetic matter $\lambda$ are given by (4.36) and (4.37) respectively. Therefore we can easily see that for $\pi$ mode the linearized approximation for constraint (5.2) remains always valid for these perturbations. For mimetic mode the last term in (5.2) starts to exceed the linear term at $L \leq m^{-\frac{3}{5}}$ when $\delta \lambda_{L}$ becomes of order $m^{2}$ and in case of mimetic matter the linear approximation fails for the constraint $\bar{h}_{0}^{0}=0$. 
Now we calculate higher order corrections to equations (4.14)-(4.17). Equation (4.14) becomes

$$
\begin{aligned}
\Delta \psi= & \lambda+\frac{m^{2}}{4}\left[(3 \psi+\Delta \pi)+\dot{\pi}_{, i} \dot{\pi}_{, i}+\frac{3}{2} \pi_{, i k} \pi_{, i k}-\frac{1}{2}(\Delta \pi)^{2}-\chi_{, i}^{0} \dot{\pi}_{, i}-\frac{1}{2} \chi_{, i}^{0} \chi_{, i}^{0}\right] \\
& +O\left(\lambda \dot{\chi}^{0}, \psi^{2}, \psi \Delta \pi, \ldots\right),
\end{aligned}
$$

where we have assumed that the gravitational potentials $\psi, \phi$ are much smaller than unity. We have neglected in (5.6) the higher order terms in metric dependence in both sides of Einstein equations as well as higher order terms such as $\psi \Delta \pi$ etc. because they are always smaller than the corresponding linear terms. Considering perturbations with wavelength $L \ll m^{-1}$ and taking into account (5.3)-(5.5) we can estimate the various quadratic terms in $(5.6)$ as

$$
\dot{\pi}_{, i} \dot{\pi}_{, i} \sim \pi_{, i k} \pi_{, i k} \sim(\Delta \pi)^{2} \sim \frac{\pi^{2}}{L^{4}} ; \quad \chi_{, i}^{0} \dot{\pi}_{, i} \sim \frac{\lambda \pi}{m L} ; \quad \chi_{, i}^{0} \chi_{, i}^{0} \sim \frac{\lambda^{2} L^{2}}{m^{2}},
$$

where we kept only the leading terms. For quantum fluctuations we have

$$
\frac{\pi^{2}}{L^{4}} \sim \frac{m^{2}}{(m L)^{4}} ; \quad \frac{\lambda \pi}{m L} \sim \frac{m^{2}}{(m L)^{7 / 2}} ; \quad \frac{\lambda^{2} L^{2}}{m^{2}} \sim \frac{m^{2}}{(m L)^{3}} .
$$

These nonlinear terms have to be compared to the linear terms in the brackets on the right hand side of equation (5.6)

$$
\Delta \pi \sim \frac{\pi}{L^{2}} \sim \frac{m}{(m L)^{2}} ; \quad \psi \sim L^{2} \lambda \sim \frac{m}{(m L)^{1 / 2}},
$$

where the first term is entirely due to the contribution of $\pi$ mode of the graviton and the main contribution to the second term comes from mimetic matter. Because $m L \ll 1$, the term $\frac{\pi^{2}}{L^{4}}$ dominates among nonlinear corrections for quantum fluctuations and is entirely due to the scalar mode $\pi$ of the massive graviton. It becomes comparable to $\Delta \pi$ at the scale

$$
L_{\pi}^{\mathrm{str}} \sim m^{-\frac{1}{2}}
$$

and for $L \leq L_{\pi}^{\text {str }}$ the scalar mode of the graviton becomes strongly coupled and decouples from the two transverse degree of freedom which, in contrast, only becomes strongly coupled at Planck scale. Among the two contributions from mimetic matter the second term in (5.8) is obviously larger than the third term and becomes comparable with the linear term $\psi$ given in (5.8) at the scale

$$
L_{\text {mim }}^{\text {str }} \sim m^{-\frac{2}{3}},
$$

where the quantized mimetic matter becomes strongly coupled. This scale is larger than the scale $\sim m^{-\frac{3}{5}}$ found above, where nonlinear corrections to the constraint $\bar{h}_{0}^{0}=0$ become important. Hence the mimetic mode gets in the strongly coupled regime when $\delta \lambda_{L}$ is yet smaller than $m^{2}$. Notice that at scale $\sim m^{-\frac{3}{5}}$ the smallest last term in (5.8) becomes of order $\psi$ given in (5.9). Equation (4.15) gets modified by the dominant nonlinear corrections as

$$
\dot{\psi}_{, i}=-\frac{m^{2}}{4}\left[\left(\chi^{0}-\dot{\pi}\right)_{, i}-\Delta \pi \chi_{, i}^{0}+2 \chi_{, k}^{0} \pi_{, i k}+\Delta \pi \dot{\pi}_{, i}-3 \pi_{, i k} \dot{\pi}_{, k}\right]+\lambda \chi_{, i}^{0}+\ldots
$$


For quantum fluctuations the nonlinear terms inside the brackets become comparable with the linear ones at $L_{\pi}^{\text {str }} \sim m^{-\frac{1}{2}}$ both for the scalar mode $\pi$ and quantum mimetic matter. The last term in this equation $\lambda \chi_{, i}^{0}$ becomes of order $m^{2} \chi_{, i}^{0}$ at scales $L \sim m^{-\frac{3}{5}}$ when the amplitude of quantum fluctuation of $\delta \lambda$ would reach the value $m^{2}$. However, as we have seen above the linearized approximation for mimetic matter fails before, at $L_{\operatorname{mim}}^{\text {str }} \sim m^{-\frac{2}{3}}$.

The higher order corrections to $i-k$ components of Einstein equations have the same structure as in (5.6) and hence do not lead to any further restrictions on the linear perturbation theory. Thus, the scalar mode of the graviton $\pi$ gets strongly coupled at length scales of order $m^{-\frac{1}{2}}$ and at smaller scales the massive graviton loses this degree of freedom.

Vector modes. For the vector perturbations equations (4.45) and (4.46), taking into account the relevant quadratic terms, become

$$
\begin{aligned}
\Delta S_{i}= & m^{2}\left[\left(S_{i}-\dot{\tilde{\chi}}_{i}\right)+2 \dot{\tilde{\chi}}_{k} \tilde{\chi}_{k, i}+\dot{\tilde{\chi}}_{k} \tilde{\chi}_{i, k}\right] \\
\dot{S}_{i, k}+\dot{S}_{k, i}= & -m^{2}\left[\left(\tilde{\chi}_{i, k}+\tilde{\chi}_{k, i}\right)+\dot{\tilde{\chi}}_{i} \dot{\tilde{\chi}}_{k}-\delta_{i k} \dot{\tilde{\chi}}_{m} \dot{\tilde{\chi}}_{m}-\tilde{\chi}_{i, m} \tilde{\chi}_{k, m}-2 \tilde{\chi}_{m, i} \tilde{\chi}_{m, k}\right. \\
& \left.-\tilde{\chi}_{i, m} \tilde{\chi}_{m, k}-\tilde{\chi}_{k, m} \tilde{\chi}_{m, i}+\delta_{i k}\left(\tilde{\chi}_{l, m} \tilde{\chi}_{l, m}+\frac{1}{2} \tilde{\chi}_{m, l} \tilde{\chi}_{l, m}\right)\right]
\end{aligned}
$$

where we have omitted the quadratic terms $S_{i}^{2}, S_{i} \dot{\tilde{\chi}}_{i}, S_{m} \tilde{\chi}_{i, m}$ because the metric perturbations $S_{i}$ are always much smaller than unity. For plane-wave perturbations with momentum $k \simeq L^{-1} \gg m$ these quadratic terms in (5.13), (5.14) can be estimated as $\frac{\tilde{\chi}^{2}}{L^{2}}$ and these become comparable with the linear terms $\frac{\tilde{\chi}}{L}$ when $\frac{\tilde{\chi}}{L} \sim 1$. For the minimal quantum fluctuations $\tilde{\chi} \sim \frac{1}{m L}$ (see (4.53)) and hence vector modes of the massive graviton come in the strong coupling regime at the same scale as the scalar mode $\pi$

$$
L_{\tilde{\chi}}^{\mathrm{str}} \sim m^{-\frac{1}{2}} .
$$

Thus, for $L \ll m^{-\frac{1}{2}}$ the massive graviton loses three out of five degrees of freedom and the two remaining transverse degrees of freedom continue to propagate as if the graviton would be massless. One can easily check that these two degrees of freedom are not influenced much by scalar and vector degrees of freedom via nonlinear corrections because the metric perturbations they induce always remain much smaller than unity. The transverse degrees of freedom become strongly coupled only at the Planck scale. When mass of the graviton vanishes, the strong coupling scale for scalar and vector modes goes to infinity and the graviton has only two propagating degrees of freedom as it must be.

\section{$6 \quad$ Static gravitational field by external source}

Let us consider the spherically symmetric field created by the mass $M$. In General Relativity the metric far away from the source (at distance $r$ ) can be written in the linear approximation as

$$
d s^{2}=(1+2 \phi) d t^{2}-(1-2 \psi) \delta_{i k} d x^{i} d x^{k}
$$

where

$$
\phi=\psi=\phi_{N}=-\frac{M}{8 \pi r},
$$

is the Newtonian gravitational potential (in units $8 \pi G=1$ ). 
In massive gravity this potential is modified at distances $r \gg m^{-1}$ where it decays exponentially fast. However, more nontrivial is that for the case of Fierz-Pauli mass term Bianchi identities inevitably enforce the scalar curvature perturbations to vanish in the linear order [5]:

$$
\delta R=2 \Delta(2 \psi-\phi)=0,
$$

from which it follows that $\phi=2 \psi$. The bending of light measures the sum of two potentials $\phi+\psi$, that must be equal to $2 \phi_{N}$. Hence, the gravitational potential $\phi$ determining for instance the motion of the planets must be in this case equal to $\frac{4}{3} \phi_{N}$, independently of the mass of the graviton, in contradiction with experiment. This is known as vDVZ discontinuity which survives in the linearized theory even in the limit when the mass of the graviton goes to zero $[16,17]$. The resolution to this apparent contradiction was found by Vainshtein who showed that the scalar mode of the massive graviton which gives extra $\frac{1}{3} \phi_{N}$ contribution to the gravitational potential $\phi$ gets strongly coupled at the scale $R_{V}$, where the linearized approximation for this mode fails and the result of General Relativity is restored in the leading order for $r<R_{V}$ [18]. Depending on the nonlinear extension of Fierz-Pauli mass term, the Vainshtein scale $R_{V}$ changes in the interval from $\left(\frac{M}{m^{4}}\right)^{\frac{1}{5}}$ to $\left(\frac{M}{m^{2}}\right)^{\frac{1}{3}}$ (see, for example, [19-21], and references there). When mass of the graviton goes to zero, Vainshtein scale grows to infinity and thus the range of scales with vDVZ discontinuity disappears.

In the theory considered here the mass term is not of FP type and vDVZ discontinuity does not arise even at linearized level. In the presence of an external source of mass $M$, among equations (4.14)-(4.18), describing the linearized scalar mode of massive graviton, only equation (4.14) gets modified as

$$
\Delta \psi=\frac{m^{2}}{4}(3 \psi+\Delta \pi)+\lambda+\frac{1}{2} T_{0}^{0},
$$

where $T_{0}^{0}=M \delta^{3}(x)$. In the consideration above this equation was used only to find $\lambda$ for a given $\psi$ and $\pi$. In turn the solution for $\psi$ and $\pi$ is given in (4.21) and (4.23) and remains unchanged. First of all let us note that $\pi$ completely decouples from mimetic matter and the gravitational field induced by a central source because $T_{0}^{0}$ comes only in combination with $\lambda$ and only in equation (4.14). Therefore we can set $\pi=0$ assuming that the constants of integration in (4.21) vanish. Then as follows from (4.23) and (4.24)

$$
\begin{aligned}
\phi & =\psi=C\left(x^{i}\right) \sin \frac{m t}{2}+D\left(x^{i}\right) \cos \frac{m t}{2} \\
& =F\left(x^{i}\right) \cos \left(\frac{m t}{2}+\alpha\left(x^{i}\right)\right) .
\end{aligned}
$$

It is interesting to note that in case of massive mimetic gravity the static solutions for the static source do not exist in the linearized version of the theory. The gravitational potential oscillates with a frequency proportional to the mass of the graviton. Of course on time scales $t \ll m^{-1}$ the potential does not change too much. Considering $t \ll m^{-1}$ and scales $r \ll m^{-1}$ we can estimate the constant of integration in (6.5) in the presence of source $T_{0}^{0}$ 
from (6.4) to get

$$
\phi=\psi \simeq-\frac{1}{8 \pi \cos \alpha}\left(\frac{M}{r}+\frac{2}{r} \int^{r} \lambda d^{3} x\right) \cos \left(\frac{m t}{2}+\alpha\right),
$$

and as follows from (4.15)

$$
\chi^{0}=-\frac{4}{m^{2}} \dot{\psi} \simeq-\frac{1}{4 \pi m \cos \alpha}\left(\frac{M}{r}+\frac{2}{r} \int^{r} \lambda d^{3} x\right) \sin \left(\frac{m t}{2}+\alpha\right)
$$

Clearly these solutions are approximate solutions valid only for $t \ll m^{-1}$. Keeping this in mind we will find the range of the scales where the linear approximation used to get them fails because of nonlinear corrections. From (5.2) and (5.6) it is clear that this happens when $\chi_{, i}^{0} \chi_{, i}^{0}$ becomes of order $\phi=\psi$. Neglecting the contribution of mimetic matter in (6.6), (6.7) and taking as a rough estimate

$$
\psi \sim \frac{M}{r}, \quad \chi_{, i}^{0} \chi_{, i}^{0} \sim \frac{\left(\chi^{0}\right)^{2}}{r^{2}} \sim \frac{M^{2}}{m^{2} r^{4}},
$$

we see that these terms become comparable at

$$
r_{\mathrm{str}} \sim\left(\frac{M}{m^{2}}\right)^{\frac{1}{3}}
$$

and for $r<r_{\text {str }}$ the nonlinear term $\chi_{, i}^{0} \chi_{, i}^{0}$ cannot be neglected. Thus the linearized approximation used to derive the non-static solutions (6.6), (6.7) is not valid for $r<r_{\text {str }}$. Moreover as follows from (5.12)

$$
\dot{\psi}_{, i}=\left(\lambda-\frac{m^{2}}{4}\right) \chi_{, i}^{0}
$$

and hence when $\lambda$ becomes comparable with $m^{2}$ the linearized $0-i$ equations fail. One can see that this equation allows static solution for $\lambda=m^{2} / 4$. In this case the contribution of mimetic matter to the gravitational potential in (6.6) becomes comparable to the contribution of the source of mass $M$ at scales $r_{\mathrm{str}} \sim\left(\frac{M}{m^{2}}\right)^{\frac{1}{3}}$.

Thus, at scales $r<r_{\text {str }}$ we find that the solution of the equation for central source is static and corresponds to

$$
\phi=\psi=\phi_{N}\left(1+O(1)\left(\frac{r}{r_{\mathrm{str}}}\right)^{3}\right),
$$

where the corrections due to the contribution of induced mimetic matter rapidly decrease towards the smaller scales. On large scales, for $m^{-1}>r>r_{\text {str }}$ the static source produces the time dependent oscillating solution

$$
\phi=\psi \simeq O(1) \phi_{N} \cos \left(\frac{m t}{2}+\alpha\right) .
$$

These time dependent oscillations are due to the induced mimetic matter which surrounds the static source. 


\section{Summary}

It is a rather interesting theoretical challenge to try to construct a theory of massive gravity free of obvious flaws in which, on Minkowski background, the graviton behaves like a massive particle. This is the main motivation for this work. Generically the five degrees of freedom of a massive graviton are accompanied by an extra ghost degree of freedom which propagates already at the linear level unless the mass term is taken to be in the Fierz-Pauli form [4]. For the FP term the dangerous mode remains non-dynamical in the linear approximation but generically reappears as nonlinear Boulware-Deser ghost [5] on non-trivial (different from Minkowski) backgrounds. There is a claim in the literature that there exists a nearly unambiguous higher order extension of the FP theory in which such ghost is absent on most of the non-trivial backgrounds [6]. However, as we have shown in $[8,10]$, the ghost field can still reappear on some backgrounds . In this paper we have addressed the following questions:

- Is it possible to have a rather simple theory of massive gravity, which remains ghost free irrespective of its nonlinear extension?

- Can we have non FP mass term and nevertheless avoid the appearance of a ghost?

As we have shown in this paper the answer to both questions is positive. The only way to generate the graviton mass without explicitly spoiling diffeomorphism invariance is to use the BEH mechanism with four scalar fields. In this case, in the broken symmetry phase, the three scalar fields donate their degrees of freedom to the graviton which thus gets a total of five degrees of freedom and acquires mass. The fourth field, if unrestricted, becomes a ghost and even for the Fierz-Pauli mass form still propagates on some nontrivial backgrounds. We have proposed to use the constrained mimetic scalar instead of this dangerous field. This field becomes dynamical and can imitate dark matter when combined with the longitudinal mode of gravity. In mimetic massive gravity its behavior is slightly modified and mimetic matter still well imitates cold dark matter. Thus, the dangerous mode is avoided and instead we get a good candidate for dark matter irrespective of the nonlinear extension of the theory. The inevitable consequence of using mimetic field, which is always in broken symmetry phase, is the need to use non Fierz-Pauli type mass term, with a relative coefficient of $-\frac{1}{2}$ between the $\bar{h}^{2}$ and $\bar{h}_{B}^{A} \bar{h}_{A}^{B}$ terms instead of -1 . Only in this case we obtain a massive graviton in the broken symmetry phase. This graviton is completely decoupled from mimetic matter in the linear approximation and VDVZ discontinuity is completely avoided. Out of the five degrees of freedom, three degrees due to the scalar fields get in the strongly coupled regime at the same scale $L^{\text {str }} \sim m^{-\frac{1}{2}}$ and at $L<L^{\text {str }}$ the graviton has only two transverse degrees of freedom which become strongly coupled at Planck scale. This is quite different from the case of FP mass term where these scales are different for the scalar and vector modes. The other interesting feature of the theory is that the gravitational potential produced by a mass $M$ is static only at scales $L<r_{\text {str }} \sim\left(\frac{M}{m^{2}}\right)^{\frac{1}{3}}$ while at larger distances it oscillates with frequency $\frac{m}{2}$ and thus vanishes, being averaged over the time scales $t>m^{-1}$. 


\section{Acknowledgments}

The work of A. H. C is supported in part by the National Science Foundation Grant No. Phys-1518371. The work of V.M. is supported in part by The Dark Universe and the Cluster of Excellence EXC 153 Origin and Structure of the Universe. V.M. thanks ENS, where a part of this work is completed, for hospitality.

Open Access. This article is distributed under the terms of the Creative Commons Attribution License (CC-BY 4.0), which permits any use, distribution and reproduction in any medium, provided the original author(s) and source are credited.

\section{References}

[1] W. Siegel, Hidden gravity in open string field theory, Phys. Rev. D 49 (1994) 4144 [hep-th/9312117] [INSPIRE].

[2] G. 't Hooft, Unitarity in the Brout-Englert-Higgs mechanism for gravity, arXiv:0708.3184 [INSPIRE].

[3] A.H. Chamseddine and V. Mukhanov, Higgs for graviton: simple and elegant solution, JHEP 08 (2010) 011 [arXiv: 1002.3877] [INSPIRE].

[4] M. Fierz and W. Pauli, On relativistic wave equations for particles of arbitrary spin in an electromagnetic field, Proc. Roy. Soc. Lond. A 173 (1939) 211 [INSPIRE].

[5] D.G. Boulware and S. Deser, Can gravitation have a finite range?, Phys. Rev. D 6 (1972) 3368 [INSPIRE].

[6] C. de Rham, G. Gabadadze and A.J. Tolley, Resummation of massive gravity, Phys. Rev. Lett. 106 (2011) 231101 [arXiv:1011.1232] [INSPIRE].

[7] A.H. Chamseddine and V. Mukhanov, Massive gravity simplified: a quadratic action, JHEP 08 (2011) 091 [arXiv: 1106.5868] [INSPIRE].

[8] L. Alberte, A.H. Chamseddine and V. Mukhanov, Massive gravity: exorcising the ghost, JHEP 04 (2011) 004 [arXiv: 1011.0183] [INSPIRE].

[9] A.H. Chamseddine and V. Mukhanov, Mimetic dark matter, JHEP 11 (2013) 135 [arXiv: 1308.5410] [INSPIRE].

[10] A.H. Chamseddine and V. Mukhanov, Hidden ghost in massive gravity, JHEP 03 (2013) 092 [arXiv: 1302.4367] [INSPIRE].

[11] A.H. Chamseddine, V. Mukhanov and A. Vikman, Cosmology with mimetic matter, JCAP 06 (2014) 017 [arXiv: 1403.3961] [INSPIRE].

[12] V. Mukhanov, Physical foundations of cosmology, Cambridge University Press, Cambridge U.K. (2005).

[13] A.H. Chamseddine and V. Mukhanov, Ghost free mimetic massive gravity, arXiv:1805.06283 [INSPIRE].

[14] V. Mukhanov and S. Winitzki, Introduction to quantum effects in gravity, Cambridge University Press, Cambridge U.K. (2007).

[15] Ya. Zel'dovich and I. Novikov, The structure and evolution of the Universe, Chicago University Press, Chicago U.S.A. (1983). 
[16] H. van Dam and M.J.G. Veltman, Massive and massless Yang-Mills and gravitational fields, Nucl. Phys. B 22 (1970) 397 [InSPIRE].

[17] V.I. Zakharov, Linearized gravitation theory and the graviton mass, JETP Lett. 12 (1970) 312 [INSPIRE].

[18] A.I. Vainshtein, To the problem of nonvanishing gravitation mass, Phys. Lett. 39B (1972) 393 [INSPIRE].

[19] N. Arkani-Hamed, H. Georgi and M.D. Schwartz, Effective field theory for massive gravitons and gravity in theory space, Annals Phys. 305 (2003) 96 [hep-th/0210184] [INSPIRE].

[20] G. Dvali, S. Hofmann and J. Khoury, Degravitation of the cosmological constant and graviton width, Phys. Rev. D 76 (2007) 084006 [hep-th/0703027] [INSPIRE].

[21] L. Alberte, A.H. Chamseddine and V. Mukhanov, Massive gravity: resolving the puzzles, JHEP 12 (2010) 023 [arXiv: 1008.5132] [INSPIRE]. 